\title{
Integrated Design of a Long-Haul Commercial Aircraft Optimized for Formation Flying
}

\author{
H.P.A. Dijkers ${ }^{1}$ and R. van Nunen ${ }^{1}$ \\ Faculty of Aerospace engineering, TU Delft, The Netherlands \\ Co-authors \\ D.A. Bos ${ }^{1}$, T.L.M. Gutleb ${ }^{1}$, L.E. Herinckx ${ }^{1}$, H. Radfar ${ }^{1}$, E. Van Rompuy ${ }^{1}$, S.E. Sayin ${ }^{1}$, J. de Wit ${ }^{1}$, and W.W.A. \\ Beelaerts van Blokland ${ }^{2}$ \\ Faculty of Aerospace engineering, TU Delft, The Netherlands
}

\begin{abstract}
The airline industry is under continuous pressure to reduce emissions and costs. This paper investigates the feasibility for commercial airlines to use formation flight to reduce emissions and fuel burn. To fly in formation, an aircraft needs to benefit from the wake vortices of the preceding aircraft. This requires a stable aerodynamic flow, accurate navigation and a highly sophisticated aircraft to counteract the negative consequences of flying in formation. It is found that the most stable region for an aircraft is between ten to twenty wingspans. For safety reasons the formation will fly in an echelon shape, indicating that only one wing of the aircraft is benefitting from the vortex. A GNSS/INS integrated navigation system is needed to allow for safe and accurate spacing between the aircraft. LiDAR based vortex detection is used to fly in the most stable and beneficial area of the vortex. Extra measures are taken to counteract the negative effects induced by flying with one wing in the vortex. A morphing wing is used to counteract the rolling moment due to an increase in lift on one wing. A strengthened tail is necessary to compensate the yaw moment induced by a reduction on drag on one wing. The benefits of formation flying in combination with the state-of-the-art open rotor jet could lead to fuel and emission savings of $54 \%$ compared to a Boeing 787. Implemented in 2030 this would be a major impact on the carbon footprint of the aviation industry.
\end{abstract}

\section{Introduction}

$\mathrm{T}$ he last few decades the oil prices have soared to above a hundred dollar per barrel, resulting in high fuel prices for aviation. This trend is continuing, so for the aerospace industry a serious problem arises, how to cut the fuel costs and become more cost effective. Furthermore, the green awareness of the public pushes the airlines to search for green alternatives to their regular kerosene fuel. As stated by the Lisbon 2020 Vision $^{1}$, the aerospace industry must decrease the emission of carbon dioxide with fifty percent by the year 2050. In addition, the overall fuel efficiency must increase with $1.5 \%$ per year, until 2020. This treaty imposes a further challenge to the aviation industry, since current development will not be able to decrease the emissions enough to reach that goal. Therefore, perhaps a step in a different direction has to be made, indicating a design change in the concept of a long haul jet aircraft.

As long as men can remember, birds fly in formation to migrate from the cold north to the warm south. This paper investigates the possibility to use the same phenomenon to reduce fuel and emissions for long haul commercial jet aircraft. This paper will show where the beneficial component of the vortex is located. How large a separation distance is needed between the airplanes for maximal benefit. The sort of navigation that is needed to allow safe and stable formation flight. And a blue print for the design of an aircraft capable of flying efficiently both solo and in formation, where special systems counteract the induced unbalance in the airplane.

This paper describes this new design, which benefits from extended formation flight. Since the working principles of extended formation flight are rather different from bird formation flight, these are explained first. Hereafter the design, specifically made to fly in formation, is discussed in the second section, and finally the overall benefit of this type of aircraft is assessed in a comparison to a modern Boeing 787.

\footnotetext{
${ }^{1}$ Bsc, Faculty of Aerospace Engineering, TU Delft, The Netherlands

2 Ass. Prof., Chair Aerospace Transport and Operations, Faculty of Aerospace Engineering, TU Delft, The Netherlands
} 


\section{Formation Flying}

The benefit of close formation flight for birds and aircraft is long recognized by aerodynamicists. Theoretical results predicted that a flock of 25 birds has a range increase of $71 \%$ compared to a single bird ${ }^{2}$. Hummel ${ }^{3,4}$ has published a number of papers over the last decades, which confirmed the earlier findings, from both classical aerodynamic theory and experiments. More recent experiments by the $\mathrm{NASA}^{5-7}$ with both unmanned aerial verhicles and manned aircraft have shown that a drag reduction of $15-20 \%$ is achievable. Recently a case study has been done on a formation flying system approach, which combines the upsides, the theoretical benefit from one aircraft, and the effectiveness of the formation of aircrafts, with the downsides of the entire system, such as distance penalties. This study indicated that up to $13 \%$ reduction in fuel consumption can be gained ${ }^{8}$. However since close formation flight is highly impractical for commercial aircraft, use is made of the persistence of trailing wake vortices. Moreover from a safety perspective, it is not feasible that commercial aircraft are flying only spans separated at cruising speed. This would pose an unacceptable risk of collision. With extended formation flight this risk is minimized, while still maintain a large part of the benefits of flying in formation. These extended formations are separated by about ten spans downstream, ensuring the safety of the aircraft. A recent study, making use of the a sophisticated wake vortex model has shown that theoretical induced drag reductions of $40 \%$ can be gained, for streamwise spacing below 50 spans 9 .

\section{Principles of extended formations}

In this paragraph the basics of extended formation are explained, as well as the theoretical principles behind it. Due to the non-uniform lift distribution over the wing of an aircraft, the initial flat wake shed from the trailing edge, rolls up into a vortex. This roll-up process takes place in the near field of the wing. In this region it is complicated to make detailed models of the wake induced velocity. For close formation flight the roll-up is therefore not accounted for, however with extended formations this leads to inadequate results. Since the wake is considered as completely rolled up after a few spans ${ }^{10}$, the separation of ten spans leaves only two completely rolled up vortices trailing from an aircraft. With this vortex structure the famous horseshoe vortex system can be introduced. This vortex system is a remarkable accurate simplification of much more complex models, in fig. 1 the trailing vortex system of an aircraft can be seen. For the scope of this paper use is made of the horseshoe vortex system.

The vortices shed from the trainling edge of the wing induce a velocity field in the surrounding air, resulting in an upwash and a downwash region. For formation flying, both close formation as well as extended formation flight, the upw ash region is used to generate the benefit. When the wing of an aircraft is

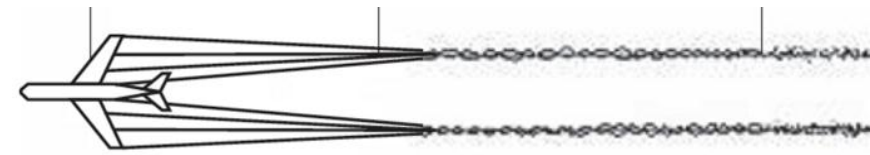

Figure 1. Trailing vortex system of an aircraft, with roll up region. positioned correctly in the trailing vortex of its predecessor, the induced drag can be reduced. This reduction of induced drag is caused by two phenomena:

- Rotation of the direction of incoming air for the wing

- Increased speed of the incoming for the wing

The rotation of the direction of the incoming air also rotates the drag and lift vector of the wing, which respectively are perpendicular and parallel to the direction vector of the incoming air of the wing. Since both the drag and lift vector are rotated a new system of forces emerges which has the effect that if the forces are decompesed in the direction perpendicular and parallel to the flight direction the induced drag is decreased in size. A side effect is that due to this rotation the lift has decreased slightly.

The increase in speed of the incoming air is also due to the induced velocity field of the vortices. The velocity component that is added to the flight velocity, causes the speed of the air to rise. This in turn causes that the lift is increased. Since the increase in lift is larger due to the speeding up of the air than the decrease due to the rotation of the direction, overall more lift is generated by the wing. Herefore the aircraft can decrease its angle of attack, since the lift can be reduced as well to balance the weight of the aircraft. This in turn causes the lift induced drag to be reduced as well, adding to the overall drag reduction.

As a consequence of flying at larger separation distances, the instabilities that are always present in a vortex become more important. The Widnall instability induces unstable waves on the vortex, for extended formations however this is of no importance ${ }^{11}$. With a cruise configuration it can be assumed that the self induced Crow motion 
of the vortex can be neglected ${ }^{12}$. In addition the atmospheric interference and the weather influence are of no importance, because part the spacing of the aircraft is below 20 spans $^{9}$, part because aircraft tend to search for stable air, with little sidewinds and preferably a low turbulence level. Vortex meandering is however a difficult process to deal with. Very little literature is available and the effects are not completely understood. In extended formations meandering and wing flutter could possibly amplify each other, if the eigenfrequency of a wing flutter mode coincides with the frequency of the vortex meandering, however this effect needs further research to be able to be conclusive about the effects or measures that have to be taken. In the scope of this paper the meandering effects are not accounted for.

In a ideal situation each aircraft in the formation would be able to benefit from two trailing vortices of two predecessors. This would ensure both wing experience almost the same aerodynamic effects and the maximal benefit can be gained. However this implies that for each aircraft to benefit two aircraft have to fly in front, possibly without benefit. Also the aircraft have to be separated correctly to avoid collisions. Next to this the two aircraft flying in front of the benefitting aircraft have to position themselves fairly accurate. Since all this has proven te be highly unpractical both wings, it is more convenient, from an operations perspective as well, to position the aircraft in an echelon formation. This operational perspective implies that the fuel burn of the aircraft has to divided as equally as possible over the formation. However having only one wing in the upwash region of a vortex leads to some complications. Namely a roll, yaw, and pitch moment are additionally induced on the aircraft. The roll moment is generated from the increase in lift on one wing, due to the velocity field of the vortex, as already explained. The yaw moment is induced from the induced drag reduction on one wing.

The pitch moment is not caused by the induced velocity field but by the flattening of the lift distribution of the wing, which is under influence of the vortex. This flattening is caused by the fact that to optimize the benefit from the vortex, the vortex core has to be positioned somewhere near the tip of the aircraft, such a flattened lift distributuion can be seen in fig. 2. So a large part of the wing flies in the upwash region, however a small part of the wing flies in the downwash region. This causes that the lift production near the tip is decreased, while the lift production on the rest of the wing is increased. This causes a more flat lift distribution over the wing. Since almost all commercial airliners fly at transonic speeds, the wings are swept back. Due to the changes in lift production a additional nose up pitch moment is induced. However initial results showed that the magnitude of this moment is rather small.

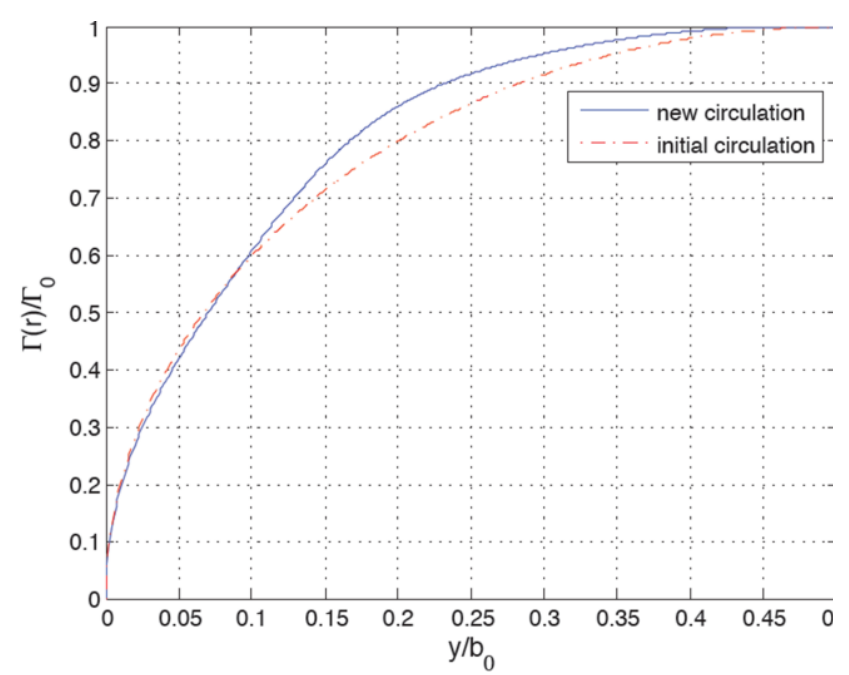

Figure 2. The lift-distribution of a wing with and without influence of a vortex

\section{Aircraft design for formation flying}

To benefit from formation flying, a new aircraft is developed to make optimal use from wake vortices and minimize the disadvantages accompanied with this. A traditional aircraft is not fit for formation flying, since the drag penalty would be too large to compensate the roll moment. The ailerons would need such a deflection that during cruise flight the drag associated with this is simply to large. Furthermore formation flying is not beneficial within a certain range. Therefore first the range, where after formation flying becomes beneficial, must be determined. Due to formation flying certain distance penalties have to be taken to allow aircraft to partially fly the same route ${ }^{13}$. In order to keep the time penalty associated with this distance penalty small, the maximal allowed penalty is about $5 \%$. With these limits the minimal range where formation flying becomes beneficial is 5,000 nautical miles. With some further market research, the capacity of the aircraft is estimated on 280 passengers, and with an optimal range of 8,000 nautical miles ${ }^{13}$. In addition, the design is made such that the implementation date is set at 2030.

The initial implementation date is set to 2030 to be able to meet the target set by the Lisbon 2020 treaty already in an early stage instead of the year 2050. Furthermore, the technology to build an aircraft fit for formation flying is still in development and will need several years to mature and be tested. So it can be expected that not all the 
technology involved is ready by the year 2030. However for the concept of the design this date is not of great importance. The technologies needed in the concept design are explained in the following paragraphs.

\section{Preliminary aircraft design}

The aircraft is designed such that it is able to fly efficiently solo as well as in formation. The aircraft has a low wing; both becausethis has a stabilizing effect while flying in formation and for normal passenger aircraft this low wing has proven to be very effective. Distortion of the wing load in sideslip gives a decreased dihedral effect and stabilizing effect for low wing. The sidewash of the vortex which is present on the wing has the same effect. The rolling moment which is caused by the cross flow will partly stabilize the roll moment created by the extra lift on the right wing, this can be seen in fig. 3. It must be noted that the sidewash on the wing is relatively small, so the stabilizing effect is small as well.
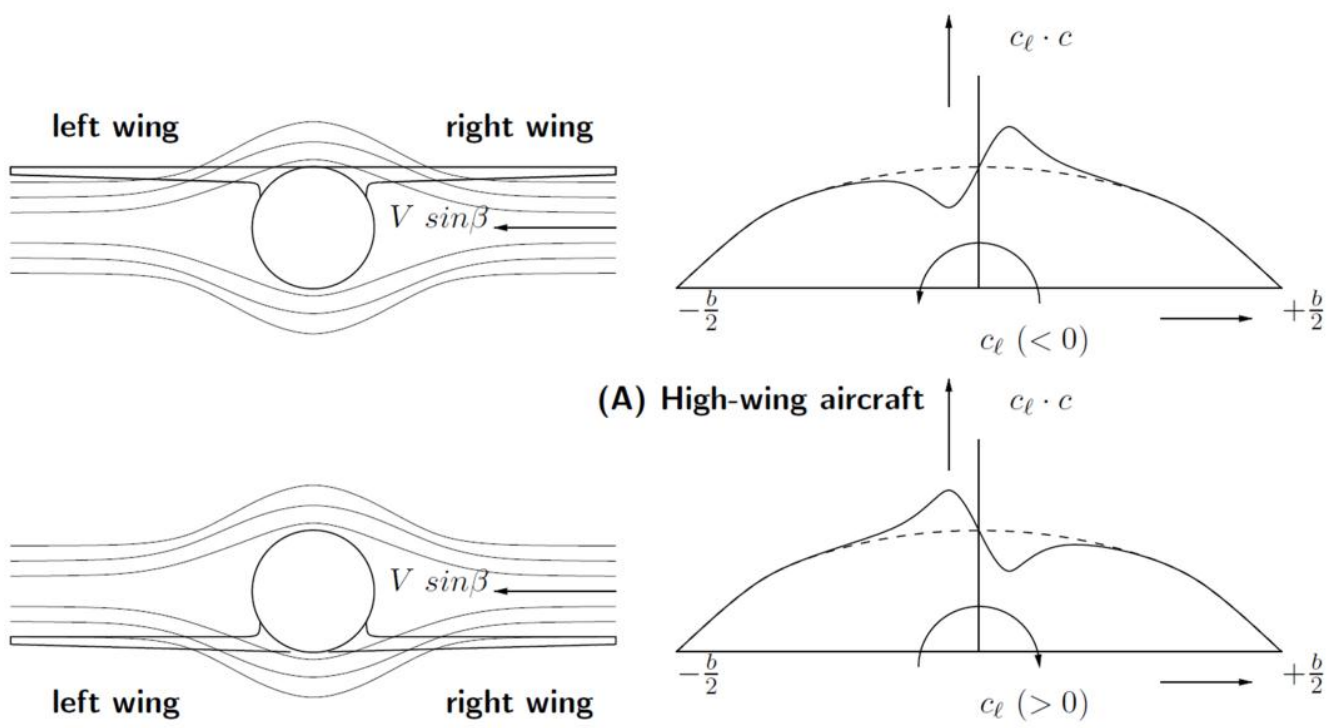

(B) Low-wing aircraft

Figure 4. Effects of a low and high wing configuration on the lift distribution of an aircraft in sideslip

The choice for the engine is made on basis of fuel consumption and efficiency. The engines are placed at the back of the fuselage to minimize interference with the wing so it can make optimal use of the vortex. The airflow that comes out of the engines could interfere with the beneficial upwash field or create turbulences in the wing structure near the wing tip. The horizontal tail is placed below the engines to allow for noise shielding. With moral obligation to reduce noise and emissions the development of engines are becoming increasingly important. Various types of new engines are planned to enter in service before the year 2030, specifically the advanced high-bypass turbofan, the geared turbofan, and the open rotor jet. The increase in efficiency of these engines has a large impact on the design of the aircraft. The less fuel the engines burn, the less fuel an airplane has to take along and thus the lighter the aircraft. The lighter the aircraft, the smaller the wings and the tails and hence less energy is needed to propel the aircraft, calling for smaller engines and thus less fuel burn. This "snowball effect" increases the importance of efficient engines.

In general, it is more efficient to give a large quantity of air a small acceleration, than give a small quantity a large acceleration, therefore increasing the bypass ratio of turbofans results in a more efficient engine. However manufacturers have been pushing the boundaries of ducted turbofans and their bypass ratios and therefore the end may well be in sight. Although an advanced turbofan can still increase its efficiency by eight to ten percent ${ }^{14}$, major breakthroughs are not expected. The geared turbofan proves significantly more promising with respect to noise reductions and fuel consumption. By introducing a gear between the low-pressure turbine and low-pressure compressor, each can rotate separately and at their optimal speed. Up until now no higher-thrust version for a widebody aircraft is announced and reliability has yet to be proven.

Therefore, the choice for the formation flyer is the ultra high bypass turbofan or the open rotor jet. The theory is that by unducting the fan a theoretical unlimited bypass ratio can be accomplished. Reduction in fuel burn of thirty percent can be expected ${ }^{15}$. Downside is that the counter rotating fan blades create a substantial amount of noise on the ground and in the cabin. General Electric ${ }^{16}$ however claims that noise standards will be met and further 
improvement is possible. The choice for the open rotor jet is mainly, because of the large increase in fuel efficiency and thereby accompanied "snowball effect".

The placing of the engines with respect to formation flight is important as well. The engines are placed at the back of the aircraft for two reasons. Firstly, allowing clean flow on the wings to make optimal use of the vortex. Secondly, the open rotor jet has a large diameter with the fan blades on the outside. To allow for ground clearance and for safety reasons, the engines are placed on the back of the aircraft. There, the horizontal tail and fuselage shields part of the noise directed to the ground. An artist impression of the aircraft is given in fig. 5 .

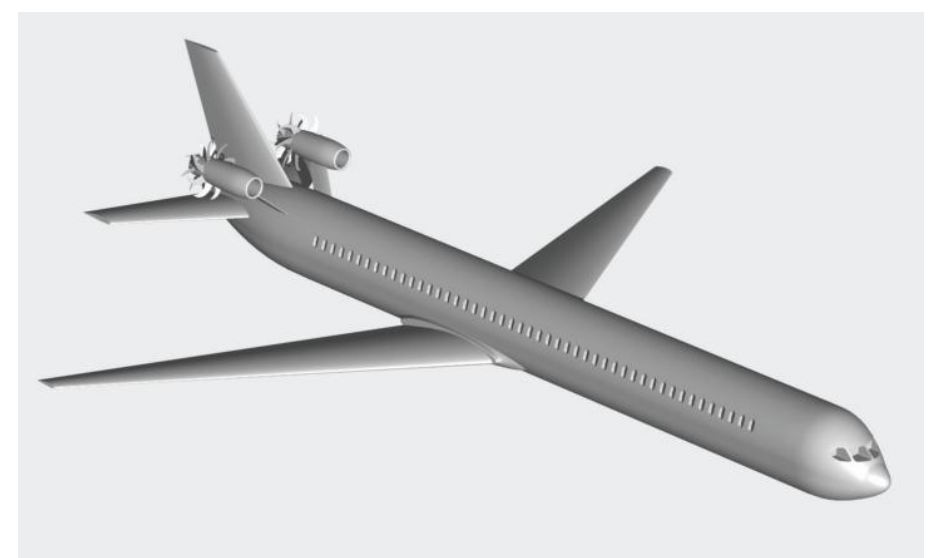

Figure 5. Artist impression of formation flying capable aircraft

\section{Formation flying systems}

In order to minimize the disadvantages of flying with one wing in a vortex specific systems are designed for the aircraft. To compensate the pitch moment, the horizontal tail needs to generate $1 \%$ more lift, leading to a negligible drag penalty.

In order to asses what type of system could be used to compensate for the roll moment a vortex lattice method has been used. With a simulation of the influence of the vortex on the wing the difference in lift between both wings is calculated to be $8 \%$. With this assessment of magnitude of the required changes the most optimal system proved to be a morphing wing structure. This allows for changing the camber of the airfoil to counteract the roll. Both the leading and trailing edge are equipped with this system. Both composites as well as aluminum alloys can be used as skin material ${ }^{14}$. Composites have the advantage that a variable stiffness matrix can be applied, which significantly improves the flexural properties of the wing in case of morphing. Since the change in camber that is required to compensate for the difference in lift is rather small, it is expected that the drag penalty is rather small.

From vortex lattice simulation it is shown that to compensate the yaw moment, the vertical tail needs to produce $14,000 \mathrm{~N}$ of lift, accompanied with a rudder deflection of 2.5 degrees. Since a normal rudder cannot cope with this force, the easiest and most efficient solution is to strengthen the tail and rudder of the aircraft. A continuous rudder deflection during cruise flight however yields a drag penalty. By using the linearized control derivatives of a horizontal tail surface one is able to asses the magnitude of this control surface drag. It is estimated that around 2 percent of the total drag of an aircraft in clean configuration is added.

\section{Navigation}

For the aircraft to fly and navigate safely in formation, additional avionics are necessary. The aircraft have to be able to position themselves accurately. This to make optimal use of the vortex of the aicraft's predecessor. For the relative positioning an integrated system of both GNSS, global navigation satellite system, and INS, inertial navigation system, is required to give enough accuracy, availability, continuity and integrity. This type of navigation is widely used in space applications. The GNSS receivers make use of carrier phase based positioning which allows for accuracy up to centimeter level ${ }^{17,18}$. Furthermore to ensure integrity monitoring, an integrated system of GNSS and INS with extended autonomous integrity monitoring is used ${ }^{19}$.

To optimally benefit from the vortex, the location of the vortex core should be accurately known. To approximate the position of the vortex, a pulsed $\mathrm{LiDAR}^{20}$ is placed on the nose of the aircraft. With the estimated vortex location, the aircraft can maneuver the wing in the vortex. To accurately position the wing two continuous wave LiDAR sensors are used ${ }^{18}$. They are placed just in front of the wing on both sides of the fuselage, which allows a field of view in a plane perpendicular to the flight direction. This results in detection accuracy up to 20 centimeter $^{18}$. An overview of position of the LiDAR sensors is given in fig. 6. 


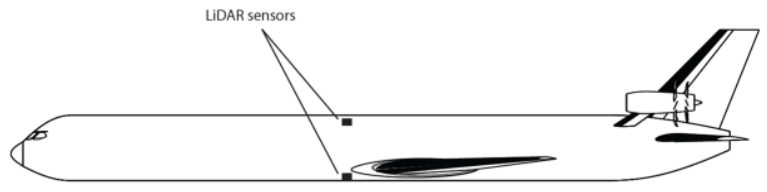

Figure 6. The location of the LiDAR sensors on the side of the aircraft.

\section{Benefit of extended formations}

\section{Simulation}

With the aircraft concept known, the actual induced drag reduction and consequent fuel reductions can be computed. For this calculation a simple flying wing model can be used. In addition, the lifting line theory is used, combined with a single bound vortex, and single trailing vortex. The influence of the second vortex of the proceeding aircraft is negligible with respect to the vortex which is positioned on the wing. This positioned trailing vortex is then imposed on the next aircraft in the formation and so forth. For the vortex a Lamb-Oseen model is used. This Gaussian velocity distribution ensures no singularities occur in the lift distribution, so the next trailing vortex can be modeled adequately.

The simulation indicated that a decrease in drag of $18 \%$ is gained, incorporating the rudder deflection. The effects of the effects of morphing on the change in lift and drag are not accounted for, since very little is known about this, especially when using supercritical airfoils. This reduction is however not present on the leader of the formation, because this aircraft does not benefit from the formation. Therefore the formation size influences the overall drag reduction gained by the formation. In fig. 7 one can see the relation between the formation size and the percentage of induced drag reduction that can be gained.

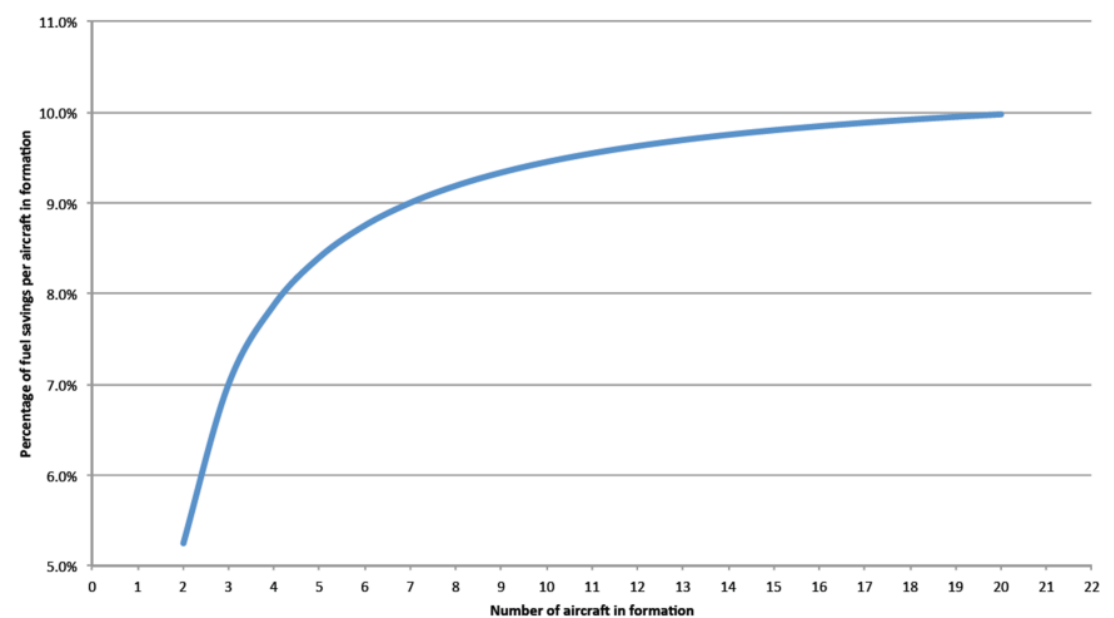

Figure 7. Graph of the percentage of fuel savings per aircraft versus the number of aircraft in formation 


\section{Comparison}

In the previously described simulation the effect of the open rotor jets was not taken into account. So for a correct comparison to be made, the additional fuel reduction of these engines is modeled as well. A realistic case is set up, with seven flights, from 4 airports in Europe, towards the east coast of America. Furthermore it is assumed that in a realistic scenario of a long-haul flight, only $67 \%$ percent can be flown in formation ${ }^{13}$. With this formation the average individual fuel consumption in the formation can be compared to the average fuel consumption of the 787 's flying to the same destinations. With this formation, the average fuel reduction is $54 \%{ }^{13}$. Therefore the goals of the Lisbon 2020 Vision are already achieved in 2030, at implementation of the aircraft concept, instead of the year 2050 .

\section{Conclusion}

For commercial aircraft to optimally benefit from extended formation flying, a new design concept is needed. In extended formations the instabilities in a vortex can be kept small by flying around 10 spans separated in flight direction. To fly efficiently in formation some new techniques are included. A normal low wing is used, since it is stabilizing in formation flight. To compensate for the roll moment induced from formation flying, a morphing wing structure is used. This structure is applied to the entire wing. The ideal material for the skin would be a variable stiffness matrix composite. Furthermore the vertical tail needs strengthening to be able to cope with the forces. A 'new' type of engine is included in the concept, reducing fuel consumption even more. Two open rotor jets are placed at the rear of the fuselage, to avoid interference with airflow over the wing. The horizontal tails provide noise shielding towards the ground, cutting the noise footprint of the aircraft. A numerical simulation indicated that a fuel reduction of $18 \%$ with formation flying alone can be gained. In a realistic case scenario with a Boeing 787 , an average reduction in fuel burn of 54\% is achievable. With this number the emissions reduction for the year 2050, set by the Lisbon 2020 Vision, is already achievable in 2030. So this new concept holds promising advantages to the aerospace industry, and has opened new fields of research.

\section{References}

${ }^{1}$ European Union, Lisbon 2020 Vision, Lisbon, Portugal, 2009.

${ }^{2}$ Lissaman, P. and Shollenberger, C., "Formation Flight of Birds," Science, Vol. 168, 1970, pp. 1003-1005.

${ }^{3}$ Hummel, D., "Aerodynamic Aspects of Formation Flight in Birds," Journal of Theoretical Biology, Vol. 104, No. 3, 1983, pp. 321-347.

${ }^{4}$ Hummel, D., "Formation Flight as an Energy-Saving Mechanism," Journal of Zoology, Vol. 41, No. 3, 1995, pp. $261-278$.

${ }^{5}$ Cobleigh, B., "Capabilities and Future Applications of the NASA Autonomous Formation Flight (AFF) Aircraft," Tech. Rep. AIAA-2002-3443, 1st UAV Conference, Portsmouth, Virginia, 2002.

${ }^{6}$ Ray, R., Cobleigh, B., Vachon, M., and John, C. S., "Flight Test Techniques used to Evaluate Performance Benefits During Formation Flight," Tech. Rep. AIAA-2002-4492, AIAA Atmospheric Flight Mechanics Conference and Exhibit, Monterey, California, 2002.

${ }^{7}$ Vachon, M., Ray, R.,Walsh, K., and Ennix, K., "F/A-18 Aircraft Performance Benefits Measured During the Autonomous Formation Flight Project," Tech. Rep. AIAA-2002-4491, AIAA Atmospheric Flight Mechanics Conference and Exhibit, Monterey, California, 2002.

${ }^{8}$ Bower, G., Flanzer, T., and Kroo, I., "Commercial Formation Flight Route Optimization," Applied Aerodynamics Conference, AIAA, 2009.

${ }^{9}$ Ning, S. A., Flanzer, T. C., and Kroo, I. M. "Aerodynamic Performance of Extended Formation Flight," $48^{\text {th }}$ AIAA Aerospace Sciences Meeting, Stanford University, Stanford, 2010.

${ }^{10}$ Widnall, S., "The structure and Dynamics of Vortex Filaments," Annual Review of Fluid Mechanics, Vol. 7, No. 1, 1975, pp. 141-165.

${ }^{11}$ Jacquin, L., Fabre, D., Sipp, D., Theofilis, V., and Vollmers, H., "Instability and unsteadiness of aircraft wake vortices," Aerospace science and technology, Vol. 7, No. 8, 2003, pp. 577-593.

${ }^{12}$ Butler, K. M., "Estimation of Wake Vortex Advection and Decay Using Meteorological Sensors and Aircraft Data," Project Report, Lincoln Laboratory, Massachusetts institute of Technology, 1993.

${ }^{13}$ Herinckx, L. E., et al., "Formation Flying as an Innovative Air Transportation System for Long-haul Commercial Flight - A Focus on Operational Feasibility and Potential Gain," Faculty of Aerospace Engineering, TU Delft, The Netherlands, 2011 (submitted for publication).

${ }^{14}$ Riegler, C. "The Geared Turbofan Technology - Opportunities, Challenges and Readiness Status”, Tech. rep., MTU Aero Engines GmbH, 2007. 
${ }^{14}$ Mul, M., “A literature study into parametric modeling of flexible cellular skin,” Faculty aerospace engineering TU Delft, Delft, The Netherlands, 2010.

${ }^{15}$ Hughes, C., "NASA Partnerships and Collaborative Research on Ultra High Bypass Cycle Propulsion Concepts", Tech rep., NASA, 2008.

${ }^{16} \mathrm{GE}$ Aviation, "Testing of Sub-Scale Blades for GE's Open Rotor Engine confirms Acoustic Qualifications". URL: http://www.geae.com/aboutgeae/pressecnter/other/other_20100719.html [cited 1 december 2010]

${ }^{17}$ Misra, P., and Enge, P., Global Positioning System - Signals, Measurements, and Performance, Ganga-Jamuna Press, Lincoln, Massachusetts, 2001.

${ }^{18}$ Verhagen, A. A., "The GNSS integer ambiguities: estimation and validation”, Ph.D. thesis, Delft University of Technology, Delft, The Netherlands, 2004.

${ }^{19}$ Hewitson, S., and Wang, J., "Extended Receiver Autonomous Integrity Monitoring (eRAIM) for GNSS/INS Integration," Journal of Surveying Engineering, Vol. 136, No. 1, 2010, pp. 13-22.

${ }^{20}$ Dolfi-Bouteyre, A., et al., "Aircraft Wake Vortex Study and Characterization with $1.5 \mathrm{~m}$ Fiber Doppler Lidar," The Onera Journal, 2009, pp 1-14. 